\title{
ISFET Based DNA Sensor: Current-Voltage Characteristic and Sensitivity to DNA Molecules
}

\author{
Lusine Gasparyan"1,2, Ilya Mazo1, Vahan Simonyan³, Ferdinand Gasparyan1,2 \\ ${ }^{1}$ Argentis LLC, Gaithersburg, MD, USA \\ ${ }^{2}$ Yerevan State University, Yerevan, Armenia \\ ${ }^{3}$ The Department of Biochemistry \& Molecular Medicine, The George Washington University, Washington DC, USA \\ Email: fgaspar@ysu.am
}

How to cite this paper: Gasparyan, L., Mazo, I., Simonyan, V. and Gasparyan, F. (2019) ISFET Based DNA Sensor: Current-Voltage Characteristic and Sensitivity to DNA Molecules. Open Journal of Biophysics, 9, 239-253.

https://doi.org/10.4236/ojbiphy.2019.94017

Received: August 7, 2019

Accepted: August 24, 2019

Published: August 27, 2019

Copyright (c) 2019 by author(s) and Scientific Research Publishing Inc. This work is licensed under the Creative Commons Attribution International License (CC BY 4.0).

http://creativecommons.org/licenses/by/4.0/

\begin{abstract}
Dependency of both source-drain current and current sensitivity of nanosize ISFET biosensor vs. concentration of DNA molecules in aqueous solution theoretically is investigated. In calculations it is carried out effects concerning charge carriers distribution in current channel and concerning carriers' mobility behavior in high electrical fields in the channel. The influence of DNA molecules on the work of ISFET biosensors is manifested by a change in the magnitude of the gate surface charge. Starting with fairly low concentrations of DNA, ISFET sensors respond to the presence of DNA molecules in an aqueous solution which is manifested by modulation of channel conductance and therefore the source-drain current changes of the field-effect transistor. It is shown that the current sensitivity with respect to concentration of DNA molecules linearly depends on the source-drain voltage and reaches high values.
\end{abstract}

\section{Keywords}

DNA, ISFET, Sensitivity, Nanosize, Current Channel

\section{Introduction}

The ion-sensitive field-effect transistor (ISFET) is one of the most popular semiconductor biosensors, and has been introduced as the first nanosized bio-chemical sensor. Currently, the use of ISFET technology encompasses a wide range of applications in a variety of areas, and those in the bioelectronic monitoring areas are particularly noteworthy. The ISFET sensor has been used to measure $\mathrm{H}^{+}$or $\mathrm{OH}^{-}$ions concentrations in aqueous solution, causing an interface potential on the gate insulator (oxide). Much attention has been paid to silicon based bio- 
sensors in the field of bio-analytical applications due to their favorable characteristics (Si-based technology, sensitivity, speed, signal-to-noise ratio, miniaturization, etc.). The introduction of the ISFET biosensor was in 1970 [1]; the first report regarding the use of an enzymatically modified ISFET for the direct detection of penicillin was in 1980 [2]. The operating principle of the ISFET device and recent advances and developments in the bio-analytical use of ISFET-based biosensors and detection strategies were covered in reviews [3] [4] [5]. Label-free detection of DNA using field-effect transistors (FET) with a real-time electrical readout system for rapid, cost-effective, and simple analysis of DNA samples has been proposed in [6]. When DNA strands bind to the free sites of gate surface of ISFETs, changes in surface electrical potential occur due to the negative charge of DNA molecule, shift in the source-drain current-voltage or capacitance-voltage characteristics thereby allowing for excellent performance of DNA sensing. Then conductivity of the semiconductor depletion layer will be modulated and source-drain signal current will be changed. It is clear that the capacitance of the system electrolyte-insulator-semiconductor depletion layer will be changed. In [7] DNA binding behavior was monitored using an ISFET biosensor, which was observed as changes in the threshold voltage. The change in DNA architecture and content, either due to hybridization or enzymatic reaction, yields a local $\mathrm{pH}$ charge variation and a rearrangement of ionic species near the sensor surface that modulate the sensor's response. One of the main disadvantages in measuring the biomolecular recognition using FET-biosensors is the Debye screening length. For this reason, it is necessary for FET measurements that biological sensing should take place within the Debye length. Field-effect transistors are described as three-electrode devices in which the current flowing between the source and drain electrodes can be modulated by changing the potential applied to the gate and source electrodes [8]. The current-control mechanism is based on an electric field generated by the voltage applied to the gate layer [8]. The current is conducted by one type of carriers (electrons or holes) depending on the semiconductor type. In the case of a p-type semiconductor, when a positive gate voltage is applied, holes are repelled from the semiconductor-insulator interface creating a depletion layer. Applying a positive gate voltage attracts electrons to the semiconductor surface, when a sufficiently high concentration of electrons is accumulated in this region a conductive channel is created at the semiconductor-insulator interface allowing a current flow between source and drain. The gate voltage modulates the channel conductance. Some groups reported on the use of FETs for real-time amplification and detection of nucleic acid using pH sensing [9] [10] [11] [12]. Detail review of advances and developments in the bio-analytical use of ISFET-based biosensors presented in [3] [5]. An electrochemical sensor for detection of unlabeled ssDNA using peptide nucleic acid probes coupled to the FET gate is demonstrated in [13]. An application of ISFET technology for the detection of single nucleotide polymorphisms suggested in [14]. In this study authors developed a useful procedure for sequencing one base via the detection of single-base mismatch in DNA. Note that 
the sequencing mechanisms of sensors based on ISFETs can actually be used only for detecting nucleic acid using $\mathrm{pH}$ sensitivity and amplifying the useful signal in real time. Static, dynamic characteristics and $\mathrm{pH}$ sensitivity of bio FET sensors made on nanosize silicon (nanowire, nanoribbon) are detailed study by us in [15] [16] [17]. In [17] $\mathrm{pH}$ sensitivity of the biochemical sensors was introduced as $\Delta I_{d s} / \Delta \mathrm{pH}$, where, $\Delta I_{d s}$ and $\Delta \mathrm{pH}$ are the elementary changes in source-drain current and $\mathrm{pH}$. Current-voltage characteristics, low-frequency noises, $\mathrm{pH}$-sensitivity and SNR (signal-to-noise ratio) for liquid and back double gated Si NW (nanowire) sensors are investigated. It is shown that source-drain current substantially depends on $\mathrm{pH}$-value and grows with increasing of the liquid gate voltage. The $\mathrm{pH}$ sensitivity increases as a result of the liquid gate voltage increase, giving opportunity to measure very low proton concentrations in the electrolyte medium at the high values of the front gate voltage. It is shown that SNR for Si NW based biochemical sensor has higher value, reaching up to $10^{5}$. In [15] [16] it is shown that in Si nanosize FET biosensors pH sensitivity increases with the increase of current channel length approaching the Nernst limit value of $59.5 \mathrm{mV} / \mathrm{pH}$, indicating that larger area devices are more suitable for the $\mathrm{pH}$ sensing. The $\mathrm{pH}$ sensitivity increases also with the increasing of the back-gate voltage and approaches to $59.5 \mathrm{mV} / \mathrm{pH}$.

The analysis conducted above shows a high level of experimental research in this area. Very good results have been achieved in the field of sensors fabrication and $\mathrm{pH}$-sensitivity; effective methods have been proposed for lowering the level of low-frequency noise in order to increase the signal-to-noise ratio. However, it seems to us that there are still many open questions when studying the physical mechanisms occurring in a semiconductor and interface semiconductor-insulator that determine and influence on the basic parameters of the sensors.

The aim of this research is the theoretical investigation, simulation and demonstration of the dependency of source-drain current of the silicon nanowire based ISFET biosensor vs. concentration of DNA molecules in aqueous solution. DNA detection mechanism and source-drain current sensitivity depending on the influence of the DNA molecules which occur in an aqueous solution over the Debye screening length will be investigated and discussed.

\section{Physical Processes in the ISFET Biosensor}

To study the source-drain current of the ISFET biosensor and its sensitivity to the presence of negatively charged DNA molecules in an aqueous solution, it is necessary to consider the physical processes occurring, in particular, at the interface between the gate insulator and the electrolyte. The main physical processes taking place in the ISFET biosensor for DNA molecule detection are sketched in Figure 1. It is presented a schematic representation of the device structure (a), distribution of the gate potential $V_{g}$ over the layered structure of the biosensor (b), and energy diagram of the electrolyte-insulator-semiconductor structure. In the left side of Figure 1 also shows the coordinate system used. 


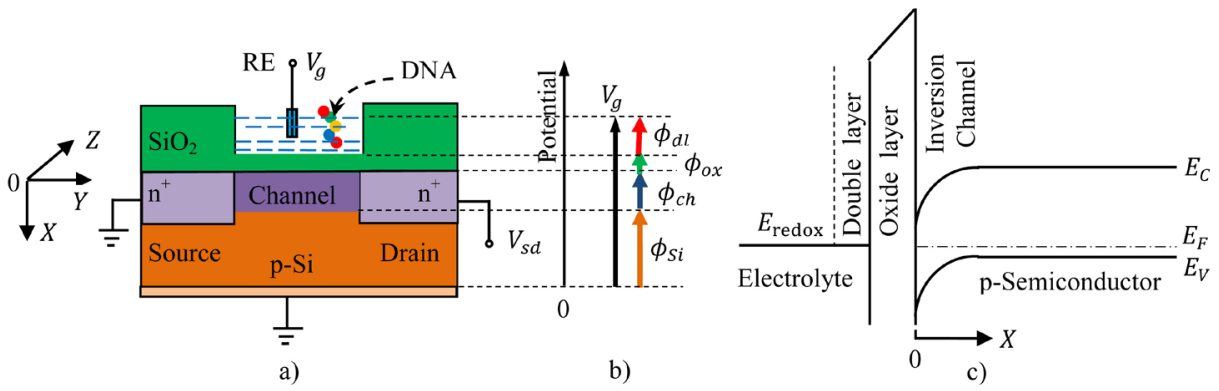

Figure 1. Schematic illustration of the ISFET biosensor (a), distribution of potentials (b), and energy diagram of ISFET in equilibrium $\left(V_{g}=0\right)(\mathrm{c})$. Here RE means reference electrode; $V_{g}$ is the gate voltage, $V_{s d}$ is the source-drain potential, $E_{F}$ is the semiconductor Fermi level energy, $E_{C}$ is the conduction and $E_{V}$ is the valence band energy, $E_{\text {redox }}$ is the redox potential. The colored circles in figure (a) show the DNA nucleotides adenine, thymine, guanine and cytosine.

Particularly silicon based structures and silicon oxide as an insulator will be discussed. In the Figure $1 \mathrm{RE}$ is the reference electrode, $V_{g}$ is the applied gate voltage, $\phi_{S i}, \phi_{c h}, \phi_{o x}$ and $\phi_{d l}$ are potentials of the silicon substrate, current channel (semiconductor depletion layer), oxide layer and double layer, correspondingly.

The balance equation for the potentials according to Figure 1, can be represented as follows:

$$
V_{g}=\phi_{s}+\phi_{S i}+\phi_{c h}+\phi_{o x}+\phi_{d l}
$$

To estimate these potentials as well as the threshold voltage, $V_{t h}$, and flat-band voltage, $V_{F B}$, we can use the following relations [8] [18]-[23]:

$$
\begin{aligned}
& V_{t h}=V_{F B}+2 \varphi_{F}+\phi_{c h} ; V_{F B}=\phi_{b u l k, s o l}-\phi_{c h}+\phi_{d l}-\frac{\Phi_{S i}-\Phi_{o x}}{q}+\frac{Q_{o x}}{C_{o x}} ; \\
& \phi_{b u l k, s o l} \approx 0 ; \quad \phi_{S i}=\phi_{b u l k, S i} \approx 0 ; \varphi_{F}=2 \varphi_{T} \ln \frac{N_{A}}{n_{i}} ; \phi_{c h}=\sqrt{\frac{4 q \varepsilon_{0} \varepsilon_{S i} N_{A} \varphi_{T}}{C_{o x}^{2}}} ; \\
& \varphi_{T}=\frac{k_{B} T}{q} ; \phi_{d l}=2 \varphi_{T}\left(\frac{\varepsilon_{w}}{\varepsilon_{r}} \frac{N_{s o l}}{K_{A K}^{+}+H_{s}^{+}}\right) ; \phi_{o x}=\frac{q N_{t}}{C_{o x}} .
\end{aligned}
$$

Here $q$ is the elementary charge; $k_{B}$ is the Boltzmann's constant; $T$ is the absolute temperature; $\varphi_{T}$ is the thermal voltage; $\varphi_{F}$ is the Fermi potential; $\phi_{b u l k, s o l}$ and $\phi_{b u l k, S i}$ are the electric potentials of the bulk solution and the bulk silicon substrate; $\phi_{d l}$ is the potential of double layer; $\Phi_{S i}$ and $\Phi_{o x}$ are the work functions of silicon and silicon oxide $\left(\mathrm{SiO}_{2}\right)$, correspondingly; $Q_{o x}$ is the oxide layer charge per unit area, $C_{o x}$ is the capacitance of the oxide layer per unit area; $\varepsilon_{0}, \varepsilon_{S i}, \varepsilon_{o x}, \varepsilon_{w}$ and $\varepsilon_{r}$ are the dielectric permittivities of free space, silicon, silicon dioxide, water and electrolyte, respectively; $N_{A}$ is the doping acceptor concentration in $\mathrm{p}$-Si substrate; $n_{i}$ is the intrinsic carrier concentration in bulk silicon; $K_{A K}^{+}$is the molar concentration of the cations in the solution, $H_{s}^{+}$is the molar concentration of the hydrogen ions at the oxide surface; $N_{s o l}$ is the molar concentration of the solution; $N_{t}$ is the concentration 
of surface open electronic binding sites (traps) per oxide unit area. Note that the redox potential $E_{\text {redox }}$ is a measure of the ease with which a molecule will accept electrons and double layer in solution consist of IHL (Inner Helmholtz layer), OHL (Outer Helmholtz layer) and GCL (Gouy-Chapman layer) [24].

The main physical processes occur in the conductive channel. Therefore, for further calculations, it is necessary to determine the surface potential of the interface between the semiconductor layer (channel) and insulator $\phi_{c h}$. This can be calculated using Equation (1) and Equation (2) and expressions for the density of minority carriers in semiconductor. For $\phi_{c h}$ we receive:

$$
\phi_{c h}=\varphi_{T} \ln \left(\frac{\eta C_{o x} \varphi_{T} N_{A}}{q t n_{i}^{2}}\right)+\varphi_{T} \ln \left\{\ln \left[1+\frac{1}{2} \exp \left(\frac{V_{g}-V_{t h}}{\eta \varphi_{T}}\right)\right]\right\} .
$$

where

$$
\eta=1+\frac{C_{d}}{C_{o x}} \approx 1+\sqrt{\frac{q \varepsilon_{0} \varepsilon_{S i} N_{A}}{2 \varphi_{T} C_{o x}^{2}}}
$$

is the factor of the transistor non-ideality $\left(C_{d}\right.$ is the capacitance of the silicon depletion layer per unit area).

We consider the case of an inversion $n$-channel liquid-gated FET (Figure $1(c))$. It is clear that the majority of processes in the structure are therefore determined by the electrons. The channel source-drain current consists of drift and diffusion components. It is well known that the diffusion component is dominant in the sub-threshold mode and the drift component is dominant in the over-threshold region. The channel source-drain current in $Y$ direction $I_{s d}(y)$ can be calculated using the following equation [8]:

$$
I_{s d}(y)=\mu_{e f} w\left[Q_{c h} \frac{\mathrm{d} V_{s d}}{\mathrm{~d} y}+\varphi_{T} \frac{\mathrm{d} Q_{c h}(y)}{\mathrm{d} y}\right] .
$$

Here $w$ is the channel width in $Z$ direction, $\mu_{e f}$ is the effective mobility and $Q_{c h}$ is the charge density of the channel mobile carriers $\left(\left[Q_{c h}\right]=\left[\mathrm{C} / \mathrm{cm}^{2}\right]\right)$ :

$$
Q_{c h}=\int_{0}^{t} q n\left(x, V_{g}\right) \mathrm{d} x .
$$

Here $t$ is the thickness of current channel in $X$ direction, $n\left(x, V_{g}\right)$ is the electron's concentration in the channel. The behavior of the source-drain current is defined by the distribution of the concentration of the mobile charge carriers over the conducting channel. Obviously, the concentration of mobile carriers in the channel depends on both the coordinate $x$ (see Figure 1(a)) and the applied gate voltage. At the same time, the charge surface concentration only depends on the gate voltage. Hence the overall concentration can be presented as follows:

$$
n\left(x, V_{g}\right)=n_{s}\left(V_{g}\right) \times f\left(x, V_{g}\right) .
$$

Here $n_{s}\left(V_{g}\right)$ is the electron surface concentration per unit area at the oxide interface and $f\left(x, V_{g}\right)$ in unit of $\left[\mathrm{cm}^{-1}\right]$ is the function which describes the charge carrier distribution in the $X$ - $Z$ plane of the channel (Figure 1) [25]. 
The surface concentration can be described using the unified charge control model from expression [26]:

$$
V_{g}-V_{t h}=\frac{q}{C_{o x}}\left(n_{s}-n_{s, t}\right)+\eta V_{t h} \ln \left(\frac{n_{s}}{n_{s, t}}\right),
$$

where $n_{s, t}$ is the surface density of electrons per unit area at the threshold voltage: $n_{s, t}=n_{s}$ at the $V_{g}=V_{t h}$. It should be noted that the influence of the charge states of the electrolyte is determined by the value of $V_{t h}$ (see Equation (2)). The concentration $n_{s, t}$ can be expressed as:

$$
n_{s, t}=\frac{\eta C_{o x} \varphi_{T}}{2 q} .
$$

Equation (7) has no analytical solution for $n_{s}$ in terms of $V_{g}$. The following approximate solution is suitable for strong inversion and sub-threshold regimes [22]:

$$
n_{s}=2 n_{s, t} \ln \left[1+\frac{1}{2} \exp \left(\frac{V_{g}-V_{t h}}{\eta \varphi_{T}}\right)\right] .
$$

After determining $n_{s}\left(V_{g}\right)$, we must also calculate the function $f\left(x, V_{g}\right)$ in order to evaluate the influence of peculiarities of the carrier distribution on the physical processes taking place in the channel.

In order to find function $f\left(x, V_{g}\right)$ for the case of the quasi classical approach, we use the following dependence of $n(x)$ [8]:

$$
n(x)=N_{c} \exp \left[-\frac{\left(E_{c}-q \phi(x)\right)-E_{F}}{k_{B} T}\right]=n_{0} \exp \left[\frac{\phi(x)}{\varphi_{T}}\right] .
$$

Here $N_{c}$ is the density of states in the conduction band of a semiconductor, $E_{c}$ is the semiconductor conduction band energy, $\phi(x)$ is the contact potential at the oxide-channel interface. To determine $\phi(x)$ we have to solve the Poisson equation:

$$
\frac{\mathrm{d}^{2} \phi(x)}{\mathrm{d} x^{2}}=-\frac{\rho(x)}{\varepsilon_{0} \varepsilon_{S i}} .
$$

Here $\rho(x)$ is the space charge density for the fully ionized acceptor centers in semiconductor (usually it is boron in silicon):

$$
\rho(x)=-q\left(N_{A}^{-}+n-p\right)=-q p_{0}\left[1-\exp \left(-\frac{\phi}{\varphi_{T}}\right)+\frac{n_{0}}{p_{0}} \exp \left(\frac{\phi}{\varphi_{T}}\right)\right] .
$$

Here $n, p$ and $n_{0}, p_{0}$ are the concentrations of the non-equilibrium and equilibrium electrons and holes, respectively, $N_{A}^{-}$is the concentration of negatively charged acceptors. We can use following boundary conditions to solve Equation (11) (see Figure 1):

$$
x \rightarrow \infty \Rightarrow \phi \rightarrow 0, \quad x \rightarrow 0 \Rightarrow \phi \rightarrow \phi_{c h} .
$$

Using Equation (12) and boundary conditions (13), we obtain the following solution of Equation (11): 


$$
\phi(x)=\left\{\phi_{c h}+\frac{q n_{0}}{\varepsilon_{0} \varepsilon_{S i}}\left[1-\exp \left(-\frac{x}{l_{s}}\right)\right]\right\} \exp \left(-\frac{x}{l_{s}}\right)
$$

where

$$
l_{s}=\frac{L_{D}}{1+n_{0} / p_{0}}, \quad L_{D}=\sqrt{\frac{\varepsilon_{0} \varepsilon_{S i} \varphi_{T}}{q p_{0}}},
$$

$L_{D}$ is the Debye screening length.

Then using expression for $\phi_{c h}$ from Equation (3) finally we have for the function $f\left(x, V_{g}\right)$ :

$$
\begin{aligned}
f\left(x, V_{g}\right)= & \frac{n_{0}}{n_{s}} \times \exp \left\{\left[\ln \left(\frac{\eta \varphi_{T} C_{o x} N_{A}}{q t n_{i}^{2}}\right)+\ln \left[\ln \left(1+\frac{1}{2} \exp \left(\frac{V_{g}-V_{t h}}{\eta \varphi_{T}}\right)\right)\right] .\right.\right. \\
& \left.\left.+\frac{q t^{2} n_{i}^{2}}{\varepsilon_{0} \varepsilon_{S i} \varphi_{T} N_{A}}\right] \exp \left(-\frac{x}{l_{s}}\right)\right\}
\end{aligned}
$$

The field caused by the applied gate voltage in the inversion layer of liquid-gated FETs changes the transport behavior of the charge carriers and results in more frequent scattering events than in the absence of the gate voltage. The carrier's mobility degrades as the result of scattering processes [27] [28] [29]. The mobility dependence on the transversal electric field ( $Y$ direction) at the applied gate voltage was taken into account using the following empiric equation [30]:

$$
\left(\mu_{e f}\right)_{x}=\mu_{0}-\theta\left(V_{g}+V_{t h}\right),
$$

where $\mu_{0}$ is the low-field magnitude of the mobility, $\theta$ is the coefficient taken as $28 \mathrm{~cm}^{2} /\left(\mathrm{V}^{2} \mathrm{~s}\right)$ [30] [31]. Since the modeling and the measurements are performed for low drain biases in linear mode, the effect of the electron velocity saturation on the drain current can be neglected. Other authors in [32] assume that when Coulomb scattering dominates the mobility dependence on the gate voltage $\mu\left(V_{g}\right)$ in the vicinity $V_{g}=V_{t h}$ can be linearized in the form [33] [34]

$$
\mu\left(V_{g}\right)=\mu_{0}+\theta\left(V_{g}-V_{t h}\right),
$$

where $\mu_{0}=\left.\mu\right|_{V_{g}=V_{t h}}, \quad \theta=\left.\frac{\mathrm{d} \mu\left(V_{g}\right)}{\mathrm{d} V_{g}}\right|_{V_{g}=V_{t h}}$, and in general case $\theta$ can be positive or negative.

In further calculations we will use expression (17) for the mobility of major carriers in the channel.

\section{Source-Drain Current}

Using Equation (4) and Equation (17) we can present the drift component of the source-drain current by following expression:

$$
I_{s d}(y) \approx \frac{q w n_{s} V_{d s}}{l}\left[\mu_{0}-\theta\left(V_{G}+V_{t h}\right)\right] \int_{0}^{t} f\left(x, V_{g}\right) \mathrm{d} x,
$$


where $n_{s}$ is determined from Equation (9).

For the integral

$$
\int_{0}^{t} f\left(x, V_{g}\right) \mathrm{d} x
$$

we have

$$
\int_{0}^{t} f\left(x, V_{g}\right) \mathrm{d} x=\frac{n_{0}}{n_{s}} \int_{0}^{t} \exp \left[G \exp \left(-\frac{x}{l_{s}}\right)\right] \mathrm{d} x \approx \frac{n_{0}}{n_{s}} t\left[1+\frac{l_{s}}{t} G\left(1-\mathrm{e}^{-t / l_{s}}\right)\right] .
$$

Here

$$
G \equiv \frac{1}{B}+\ln B+\ln \left[\ln \left(1+\frac{1}{2} \exp \left(\frac{V_{g}-V_{t h}}{\varphi_{T}}\right)\right)\right], \quad B \equiv \frac{\varphi_{T} \varepsilon_{0} \varepsilon_{o x} N_{A}}{q t^{2} n_{i}^{2}}
$$

Thus

$$
I_{s d}(y) \approx q w n_{0} V_{d s} \frac{t}{l}\left[\mu_{0}-\theta\left(V_{G}+V_{t h}\right)\right]\left[1+\frac{l_{s}}{t} G\left(1-\mathrm{e}^{-t / l_{s}}\right)\right] .
$$

Behavior of dependency $I_{s d}$ from number of DNA molecules in solution $N_{\text {DNA }}$ can be determined from dependency $V_{t h}\left(Q_{o x}\right)$.

For simplicity of further calculations assume that $\eta \approx 1$ and taking account that oxide layer capacitance for unit area

$$
C_{o x}=\frac{\varepsilon_{0} \varepsilon_{o x}}{t} .
$$

For $V_{t h}$ from (2) we have:

$$
V_{t h}=2 \varphi_{F}+\phi_{d l}-\frac{\Phi_{S i}-\Phi_{o x}}{q}+\frac{Q_{o x}}{C_{o x}} .
$$

Substituting (23) into (21) gives:

$$
\begin{aligned}
& I_{s d}\left(Q_{o x}\right) \approx q w n_{0} V_{d s} \frac{t}{l}\left[\mu_{0}-\theta\left(V_{G}+2 \varphi_{F}+\phi_{d l}-\frac{\Phi_{S i}-\Phi_{o x}}{q}+\frac{Q_{o x}}{C_{o x}}\right)\right] \\
& \times\left\{1+\frac{l_{s}}{t}\left\{\frac{1}{B}+\ln B+\ln \left[\ln \left(1+\frac{1}{2} \exp \left(\frac{V_{g}+2 \varphi_{F}+\phi_{d l}-\frac{\Phi_{S i}-\Phi_{o x}}{q}+\frac{Q_{o x}}{C_{o x}}}{\varphi_{T}}\right)\right)\right]\right\}\left(1-\mathrm{e}^{-t / l_{s}}\right)\right\} .
\end{aligned}
$$

The influence of the oxide interface on the source-drain current of the transistor can be taken into account by the change of the charge of the oxide interface traps $Q_{o x}=q N_{t} \quad\left(N_{t}\right.$ is the surface concentration of oxide interface all proton donors and proton acceptors traps in units $\mathrm{cm}^{-2}$, Figure 2). Depending on presence of negatively charged DNA molecules the charge on the oxide surface will decrease (compensated) and become

$$
Q_{o x}^{\prime}=q N_{t}^{+}(1-\delta), \delta \equiv \frac{N_{\mathrm{DNA}}}{N_{t}^{+}},
$$

where $N_{t}^{+}$and $N_{\text {DNA }}$ are the surface concentrations of positively charged proton acceptor $\mathrm{OH}_{2}^{+}$traps (Figure 2) and DNA surface concentration in solution 


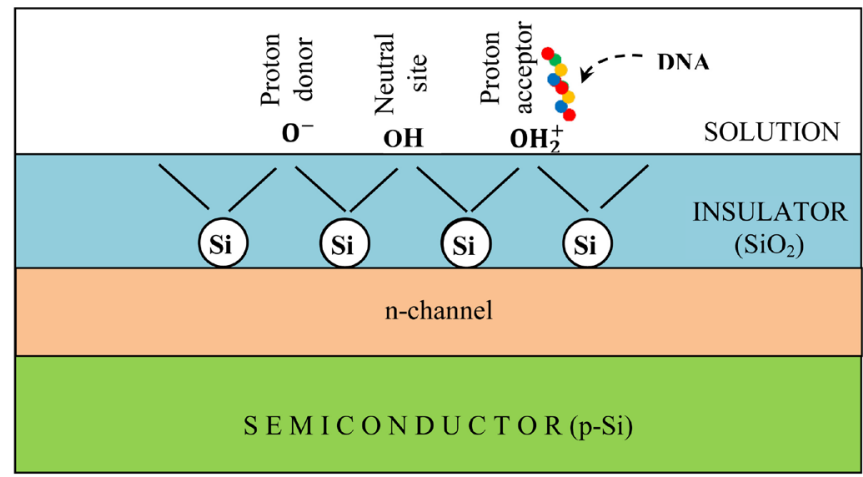

a)

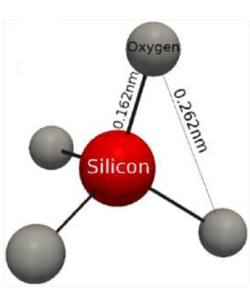

b)

Figure 2. Site binding on the interface $\mathrm{SiO}_{2}$-solution and the process of binding of the negatively charged DNA on the $\mathrm{OH}_{2}^{+}$free bond (a), $\mathrm{SiO}_{2}$ tetrahedron structure (b).

near the oxide at a distance of the Debye length. It is clear that (25) is correct for the $N_{\mathrm{DNA}} \leq N_{t}^{+}$, or $\delta \leq 1$. In the case of super compensation when $N_{\mathrm{DNA}}>N_{t}^{+}$ the DNA additional molecules do not bind on the surface positively charged sites (proton acceptors, Figure 2) and ISFET biosenor do not sense this DNA additional molecules.

Figure 2 show site binding on the interface $\mathrm{SiO}_{2}$-solution, process of negatively charged DNA molecule binding and silicon dioxide tetrahedron structure.

Consider the source-drain current sensitivity of the ISFET biosensor to DNA molecules $S$ as a change in source-drain current $\Delta I_{s d}$ for a corresponding change in the proportion of DNA molecules in the solution $\Delta \delta$ :

$$
S=\left|\frac{\Delta I_{s d}}{\Delta \delta}\right|, \mathrm{A}
$$

As $\Delta \delta$ is dimensionless parameter sensitivity will be measured by the Ampere.

\section{Numerical Simulation and Discussion}

For numerical computation, we use the following values, which correspond to the sample geometry and the parameters of the materials for the investigated nanosize structure at the room temperature: $\mu_{0}=260 \mathrm{~cm}^{2} /(\mathrm{V} \cdot \mathrm{s})$ [35], $\theta=28 \mathrm{~cm}^{2} /\left(\mathrm{V}^{2} \cdot \mathrm{s}\right) \quad$ [30] [31], $\varphi_{T}=0.026 \mathrm{~V}, N_{\text {sol }}=0.015 \mathrm{~mol} / 1 \quad$ [25], $K_{A K}^{+}=0.001 \mathrm{~mol} / 1$ [25], $w=150 \mathrm{~nm}, l=200 \mathrm{~nm}, t=20 \mathrm{~nm}, \quad \varepsilon_{S i}=11.6$, $\varepsilon_{o x}=3.9, \quad \varepsilon_{w} \approx 80, \quad \varepsilon_{r} \approx 78, \quad \varepsilon_{0}=8.85 \times 10^{-14} \mathrm{~F} / \mathrm{cm}, \quad q=1.6 \times 10^{-19} \mathrm{C}$, $\Phi_{S i}=4.85 \mathrm{eV} \quad[36], \quad \Phi_{o x}=5 \mathrm{eV} \quad[36], N_{A}=10^{15} \mathrm{~cm}^{-3}, N_{V}=2.5 \times 10^{19} \mathrm{~cm}^{-3}$ $\left(m_{n}^{*}=m_{p}^{*} \equiv m_{0}=9.1 \times 10^{-31} \mathrm{~kg}, m_{n}^{*}\right.$ and $m_{p}^{*}$ are effective mass of electrons and holes, $m_{0}$ is the free electron mass), $p_{0} \approx N_{A}=10^{15} \mathrm{~cm}^{-3}$. Let's consider the case when "power of hydrogen" of the solution is equal to 7 ( $\mathrm{pH}=7$ ). As

$$
\mathrm{pH}=-\log \left[\mathrm{H}^{+}\right] \text {, }
$$

we get

$$
\left[\mathrm{H}^{+}\right]=10^{-\mathrm{pH}}=10^{-7} \mathrm{~mol} / 1 .
$$

For the electron concentration in the inversion layer we can assume that it is 
equal to majority carrier's concentration in p-Si, e.g. $n_{0}($ inv $) \approx p_{0}=10^{15} \mathrm{~cm}^{-3}$. For the $N_{t}^{+}$we can do following estimation. It is assumed that traps concentration on the interface silicon oxide-electrolyte is the same as silicon oxide-Si. According to data [36] traps concentration in $\mathrm{Si}_{-} \mathrm{SiO}_{2}$ interface is about $\left(10^{10}-10^{11}\right) \mathrm{cm}^{-2}$. In further calculation we will use $N_{t}^{+} \approx 10^{11} \mathrm{~cm}^{-2}$.

For numerical simulation let's simplify expression for source-drain current assuming that:

1) At the noted above parameters $B \propto 10^{10}$ and we can ignore term $\frac{1}{B}$ compared $\ln B$ as $\frac{1}{B} \ll \ln B$;

2)
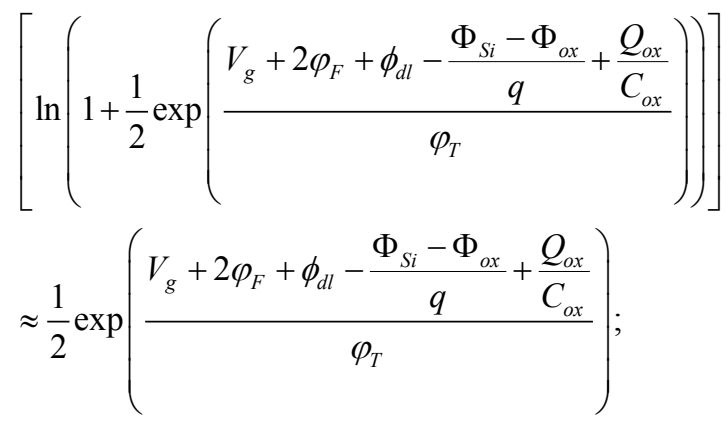

3)

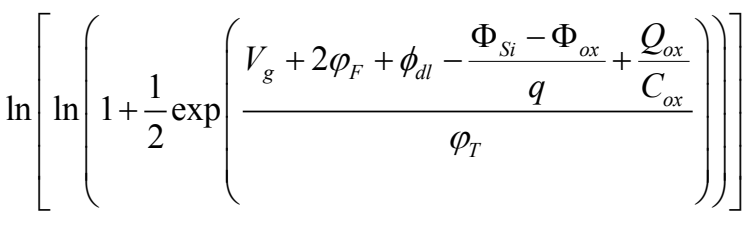

$$
\approx \ln \frac{1}{2} \exp \left(\frac{V_{g}+2 \varphi_{F}+\phi_{d l}-\frac{\Phi_{S i}-\Phi_{o x}}{q}+\frac{Q_{o x}}{C_{o x}}}{\varphi_{T}}\right) ;
$$

4) $1-\mathrm{e}^{-t / l_{s}} \approx 1-\frac{t}{l_{s}}$.

Thus source-drain current can be presented as follows:

$$
\begin{aligned}
I_{s d} \approx & \frac{q t w n_{0} V_{d s}}{l \varphi_{T}}\left[\mu_{0}-\theta\left(V_{G}+2 \varphi_{F}+\phi_{d l}-\frac{\Phi_{S i}-\Phi_{o x}}{q}+\frac{Q_{o x}}{C_{o x}}\right)\right] \\
& \times\left[\varphi_{T}\left(1+\ln \frac{B}{2}\right)+V_{g}+2 \varphi_{F}+\phi_{d l}-\frac{\Phi_{S i}-\Phi_{o x}}{q}+\frac{Q_{o x}}{C_{o x}}\right] .
\end{aligned}
$$

Results of numerical calculations of source-drain current $I_{s d}$ vs source-drain voltage $V_{d s}$ and DNA concentration are presented in Figures 3-5. The error in plotting the dependencies in Figures 3-5 does not exceed (5 - 7)\%. In order not to complicate the graphs, these errors are not shown in the figures. Note that fluctuations of values by $(5-7) \%$ particularly do not affect the course of dependencies and do not change the mechanisms for explaining their behavior. As expected, the dependence of $I_{s d}$ on the source-drain voltage is linear. Dependency $I_{s d}$ vs DNA concentration $N_{\text {DNA }}$ (or $\delta$ ) is very weak (see Figure 4). At the beginning $(0<\delta<0.1)$ source-drain current increases very weakly. This 


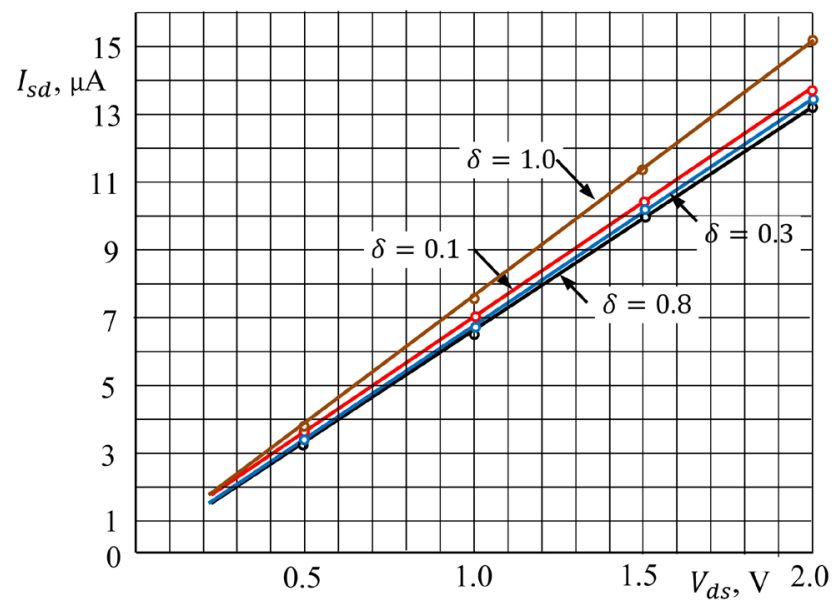

Figure 3. Dependency of the source-drain current vs source-drain voltage.

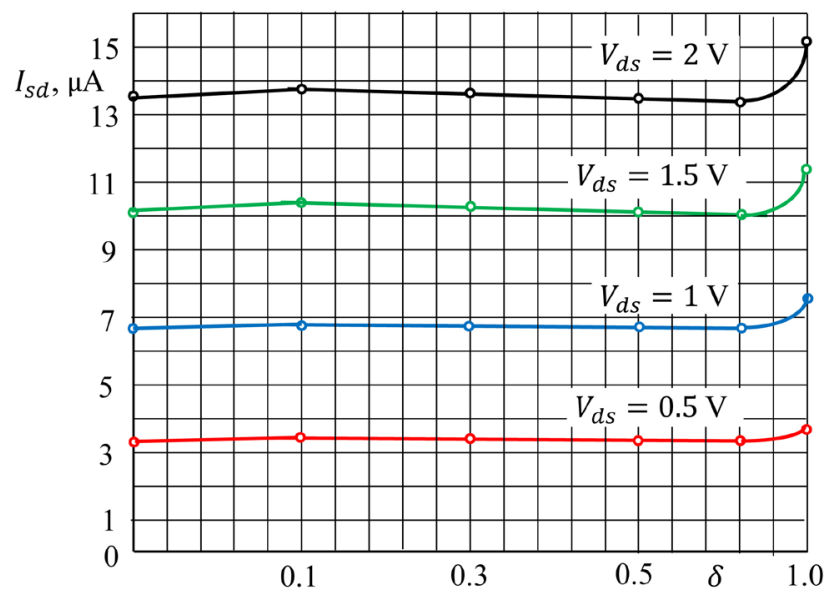

Figure 4. Dependency of the source-drain current vs DNA molecules concentration in solution.

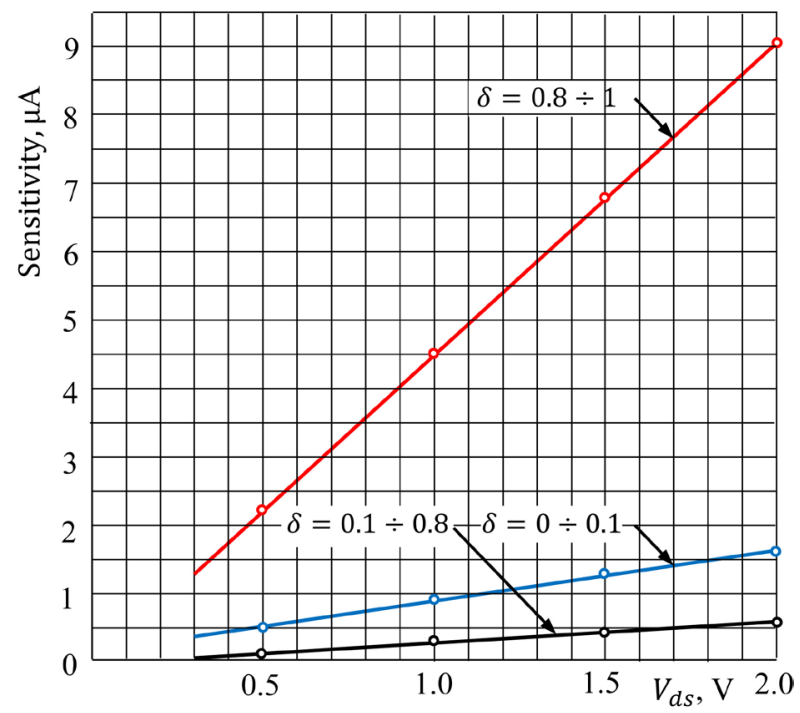

Figure 5. Dependency of the current sensitivity vs. source-drain voltage at the several values of DNA molecules concentration $N_{\mathrm{DNA}}$ or $\delta$. 
behavior can be explained as follows. Assume that all proton donors $\left(\mathrm{OH}^{-}\right)$sites in the interface oxide-electrolyte (Figure 2) fully accept protons $\left(\mathrm{H}^{+}\right)$from solution and changes in their charges are not significant and can be neglected during the sensor operation. At low concentration of DNA molecules $0<\delta<0.1$, they are bind very weakly or not bind with free proton acceptor sites $\mathrm{OH}_{2}^{+}$due to its high diffusion activity and the presence of a Coulomb barrier near proton acceptor sites. With increasing DNA concentration and therefore decreasing their diffusion activity (when $0.1<\delta<0.8$ ) negatively charged DNA molecules overcome Coulomb barrier near the proton acceptor sites and bond with them on the oxide surface. As a result the positive surface charge decreases and correspondingly decreases deepness of current channel and its conductivity (source-drain current). At the super compensation of the proton acceptor sites $\mathrm{OH}_{2}^{+}(\delta \rightarrow 1$, high concentration of DNA molecules) in oxide-electrolyte interface $I_{s d}(\delta)$ dependency has increasing behavior. Probably it is conditioned by the ionic Coulomb blockade effect [37]. As it is known this effect appears in the electro-diffusive transport of charged ions (in our case DNA molecules) through sub-nanometer artificial nanopores [38] or biological ion channels [39]. DNA molecules cannot overcome Coulomb barrier of the proton acceptor sites and channel conductance again increase. Consequently, the current increases.

The dependence of current sensitivity on source-drain voltage for several values of DNA molecules concentration is presented in Figure 5. These dependencies are constructed according to formula (26) using the data in Figure 4. They are also linear dependency. Depending on values of DNA concentration in solution and source-drain applied voltage current sensitivity can reach comparatively high values (up to $5 \ldots 9 \mu \mathrm{A} / \delta$ in the case of parameters chosen above).

Based on the above reasoning, we can draw the following conclusions.

$\checkmark$ ISFET nanosized structures can be used for detecting charged DNA molecules.

$\checkmark$ The influence of DNA molecules on the work of ISFET biosensors is manifested by a change in the magnitude of the surface charge of the gate electrode.

$\checkmark$ Starting with fairly low concentrations of DNA, ISFET biosensors respond to the presence of DNA molecules in an aqueous solution which is manifested by modulation of channel conductance or the source-drain current.

$\checkmark$ Current sensitivity linearly depends on the source-drain voltage and reaches high values.

Thus, ISFET nanosized silicon biosensors can be successfully used to detect very low concentrations of DNA molecules in an aqueous solution with high sensitivity. As an advantage note also the compatibility of silicon based devices with modern CMOS technology.

\section{Conflicts of Interest}

The authors declare no conflicts of interest regarding the publication of this paper. 


\section{References}

[1] Bergveld, P. (1970) Development of an Ion Sensitive Solid State Device for Neurophysiological Measurements. IEEE Transactions on Biomedical Engineering, BME-17, 70-71. https://doi.org/10.1109/TBME.1970.4502688

[2] Caras, S. and Janata, J. (1980) Field Effect Transistor Sensitive to Penicillin. Analytical Chemistry, 52, 1935-1937. https://doi.org/10.1021/ac50062a035

[3] Lee, C.-S., Kim, S.K. and Kim, M. (2009) Ion-Sensitive Field-Effect Transistor for Biological Sensing. Sensors, 9, 7111-7131. https://doi.org/10.3390/s90907111

[4] Veigas, B., Fortunato, E. and Baptista, P.V. (2015) Field Effect Sensors for Nucleic Acid Detection: Recent Advances and Future Perspectives. Sensors, 15, 10380-10398. https://doi.org/10.3390/s150510380

[5] Hu, Y. (2015) Advanced Sensing and Processing Methodologies for ISFET Based DNA Sequencing. Imperial College London, London.

[6] Ingebrandt, S., Han, Y., Nakamura, A., Poghossian, A., Schöning, M.J. and Offenhausser, A. (2007) Label-Free Detection of Single Nucleotide Polymorphisms Utilizing the Differential Transfer Function of Field-Effect Transistors. Biosensors and Bioelectronics, 22, 2834-2840. https://doi.org/10.1016/j.bios.2006.11.019

[7] Goncalves, D., Prazeres, D., Chu, V. and Conde, J. (2008) Detection of DNA and Proteins Using Amorphous Silicon Ion-Sensitive Thin-Film Field Effect Transistors. Biosensors and Bioelectronics, 24, 545-551.

[8] Sze, S.M. (1986) Physics of Semiconductor Devices. Wiley, New York.

[9] Veigas, B., Branquinho, R., Pinto, J.V., Wojcik, P.J., Martins, R., Fortunato, E. and Baptista, P.V. (2014) Ion Sensing (EIS) Real-Time Quantitative Monitorization of Isothermal DNA Amplification. Biosensors and Bioelectronics, 52, 50-55. https://doi.org/10.1016/j.bios.2013.08.029

[10] Wong, M.L. and Medrano, J.F. (2005) Real-Time PCR for mRNA Quantitation. BioTechniques, 39, 75-85. https://doi.org/10.2144/05391RV01

[11] Branquinho, R., Veigas, B., Pinto, J.V., Martins, R., Fortunato, E. and Baptista, P.V. (2011) Real-Time Monitoring of PCR Amplification of Proto-Oncogene c-MYC Using a $\mathrm{Ta}_{2} \mathrm{O}_{5}$ Electrolyte-Insulator-Semiconductor Sensor. Biosensors and Bioelectronics, 28, 44-49. https://doi.org/10.1016/j.bios.2011.06.039

[12] Toumazou, C., Shepherd, L., Reed, S., Chen, G., et al. (2013) Simultaneous DNA Amplification and Detection Using a $\mathrm{pH}$-Sensing Semiconductor System. Nature Methods, 10, 641-646.

[13] Kaisti, M., Kerko, A., Aarikka, E., Saviranta, P., Boeva, Z., Soukka, T. and Lehmusvuori, A. (2017) Real-Time Wash-Free Detection of Unlabeled PNA-DNA Hybridization. Scientific Reports, 7, Article No. 15734. https://doi.org/10.1038/s41598-017-16028-7

[14] Purushothaman, S., Toumazou, C. and Ou, C. (2006) Protons and Single Nucleotide Polymorphism Detection: A Simple Use for the Ion Sensitive Field Effect Transistor. Sensors and Actuators B: Chemical, 114, 964-968. https://doi.org/10.1016/j.snb.2005.06.069

[15] Gasparyan, F., Zadorozhnyi, I., Khondkaryan, H., Arakelyan, A. and Vitusevich, S. (2018) Photoconductivity, pH Sensitivity, Noise, and Channel Length Effects in Si Nanowire FET Sensors. Nanoscale Research Letters, 13, 87-96. https://doi.org/10.1186/s11671-018-2494-5

[16] Gasparyan, F., Zadorozhnyi, I., Khondkaryan, H., Arakelyan, A. and Vitusevich, S. (2017) Biochemical Sensors Based on Silicon Nanoribbon FETs. Part 1: Samples 
Fabrication, CVCs, pH-Sensitivity. Proceedings of 11 th International Conference on Semiconductor Micro- \& Nanoelectronics, Yerevan, Armenia, 23-25 June 2017.

[17] Gasparyan, F.V. and Khondkaryan, H.D. (2015) Low-Frequency Noises and SNR of Double Gated Si NW ISFET Based Biochemical Sensor. Proceedings of 10 th International Conference on Semiconductor Micro- \& Nanoelectronics, Yerevan, Armenia, 11-13 September 2015, 68-71.

[18] Gasparyan, F.V., Poghossian, A., Vitusevich, S.A., Petrychuk, M.V., Sydoruk, V.A., Siqueira, J.R., Oliveira, O.N., Offenhäusser, A. and Schöning, M.J. (2011) Low-Frequency Noise in Field-Effect Devices Functionalized with Dendrimer/Carbon-Nanotube Multilayers. IEEE Sensors Journal, 11, 142-149. https://doi.org/10.1109/ISEN.2010.2052355

[19] Deen, M.J., Shinwari, M.W., Ranuárez, J.C. and Landheer, D. (2006) Noise Considerations in Field-Effect Biosensors. Journal of Applied Physics, 100, Article ID: 074703. https://doi.org/10.1063/1.2355542

[20] Hassibi, A., Navid, R., Dutton, R.W. and Lee, T.H. (2004) Comprehensive Study of Noise Processes in Electrode Electrolyte Interfaces. Journal of Applied Physics, 96, 1074-1082. https://doi.org/10.1063/1.1755429

[21] Janicki, M., Daniel, M., Szermer, M. and Napieralski, A. (2004) Ion Sensitive Field Effect Transistor Modelling for Multidomain Simulation Purposes. Microelectronics Journal, 35, 831-840. https://doi.org/10.1016/j.mejo.2004.06.015

[22] Ytterdal, T., Cheng, Y. and Fjeldly, T.A. (2003) Device Modeling for Analog and RF CMOS Circuit Design. John Wiley \& Sons, New York.

https://doi.org/10.1002/0470863803

[23] Tarasov, A., Wipf, M., Stoop, R.L., Bedner, K., Fu, W., Guzenko, V.A., Knopfmacher, O., Calame, M. and Scho, C. (2012) Understanding the Electrolyte Background for Biochemical Sensing with Ion-Sensitive Field-Effect Transistors. ACS Nano, 6, 9291-9298. https://doi.org/10.1021/nn303795r

[24] Nakamura, M., Sato, N., Hoshi, N. and Sakata, O. (2011) Outer Helmholtz Plane of the Electrical Double Layer Formed at the Solid Electrode-Liquid Interface. ChemPhysChem, 12, 1430-1434. https://doi.org/10.1002/cphc.201100011

[25] Pud, S., Gasparyan, F., Petrychuk, M., Li, J., Offenhausser, A. and Vitusevich, S.A. (2014) Single Trap Dynamics in Electrolyte-Gated Si-Nanowire Field Effect Transistors. Journal of Applied Physics, 115, Article ID: 233705. https://doi.org/10.1063/1.4883757

[26] Gao, X.P.A., Zheng, G. and Lieber, C.M. (2010) Subthreshold Regime has the Optimal Sensitivity for Nanowire FET Biosensors. Nano Letters, 10, 547-552. https://doi.org/10.1021/nl9034219

[27] Sabnis, A.G. and Clemens, J.T. (1979) Characterization of the Electron Mobility in the Inverted (100) Si Surface. International Electron Devices Meeting, 25, 18-21. https://doi.org/10.1109/IEDM.1979.189528

[28] Woo, J. and Cheng, B. (1996) Measurement and Modeling of the N-Channel and P-Channel MOSFET's Inversion Layer Mobility at Room and Low Temperature Operation. Journal de Physique IV Colloque, 6, C3-43-C3-47.

[29] Zhou, X. and Lim, K.Y. (2000) A Novel Approach to Compact I-V Modeling for Deep-Submicron MOSFET's Technology Development with Process Correlation. Technical Proceedings of the International Conference on Modeling and Simulation of Microsystems (MSM2000), San Diego, CA, 27-29 March 2000, 333-336.

[30] Park, C., Lee, C., Lee, K., Moon, B.-J., Byun, Y.H. and Shur, M. (1991) A Unified Current-Voltage Model for Long-Channel nMOSFETs. IEEE Transactions on Elec- 
tron Devices, 38, 399-406. https://doi.org/10.1109/16.69923

https://www.academia.edu/23535536/A unified current-voltage model for long-c hannel nMOSFETs

[31] Gildenblat, G.S. and Huang, C.-L. (1989) Engineering Model of Inversion Channel Mobility for 60-300k Temperature Range. Electronics Letters, 25, 634-636. https://doi.org/10.1049/el:19890430

[32] Rudenko, T., Kylchitska, V., Khairuddin, M. Arshad, M., Raskin, J.-P., Nazarov, A. and Flandre, D. (2011) On the MOSFET Threshold Voltage Extraction by Transconductance and Transconductance-to-Current Ratio Change Methods: Part I-Effect of Gate-Voltage-Dependent Mobility. IEEE Transactions on Electron Devices, 58, 4172-4178. https://doi.org/10.1109/TED.2011.2168226

[33] Takagi, S., Iwase, M. and Toriumi, A. (1988) On the Universality of Inversion-Layer Mobility in N- and P-Channel MOSFETs. Technical Digest, International Electron Devices Meeting, San Francisco, CA, 11-14 December 1988, 398-401. https://doi.org/10.1109/IEDM.1988.32840

[34] Tsuno, M., Suga, M., Tanaka, M., Shibahara, K., Miura-Mattausch, M. and Hirose, M. (1999) Physically-Based Threshold Voltage Determination for MOSFETs of All Gate Lengths. IEEE Transactions on Electron Devices, 46, 1429-1434. https://doi.org/10.1109/16.772487

[35] Jeong, C., Antoniadis, D. and Lundstrom, M.S. (2009) On Backscattering and Mobility in Nanoscale Silicon MOSFETs. IEEE Transactions on Electron Devices, 56, 2762-2769. https://doi.org/10.1109/TED.2009.2030844

[36] Pantelides, S.T., Wang, S., Franceschetti, A., et al. (2006) $\mathrm{Si} / \mathrm{SiO}_{2}$ and $\mathrm{SiC} / \mathrm{SiO}_{2}$ Interfaces for MOSFETs-Challenges and Advances. Materials Science Forum, 527-529, 935-948. https://doi.org/10.4028/www.scientific.net/MSF.527-529.935

[37] Krems, M. and Di Ventra, M. (2013) Ionic Coulomb Blockade in Nanopores. Journal of Physics: Condensed Matter, 25, Article ID: 065101. https://doi.org/10.1088/0953-8984/25/6/065101

[38] Feng, J., Liu, K., Graf, M., Dumcenco, D., Kis, A., Di Ventra, M. and Radenovich, A. (2016) Observation of Ionic Coulomb Blockade in Nanopores. Nature Materials, 15, 850-855. https://doi.org/10.1038/nmat4607

[39] Kaufman, I.K., McClintock, P.V.E. and Eisenberg, R.S. (2015) Coulomb Blockade Model for Permeation and Selectivity in Biological Ion Channels. New Journal of Physics, 17, Article ID: 083021. https://doi.org/10.1088/1367-2630/17/8/083021 